\title{
A Case Report of Toxicity to an Opioid Analgesic
}

\section{BHARAT SHARMA ${ }^{*_{1}}$, BHAVNA GUPTA²}

Opioid synthetic pain killers act centrally and are commonly used for management of high grade pain. Although it is a standard drug being prescribed all over the world, its accidental or intentional overdose is more common in adolescent and adult males. We report the case of a 7-year-old boy who was brought to our clinic in an unconscious state following opioid intoxication. He was managed with conservative approach and made a significant improvement before discharge.

KEYWORDS: Toxicity, Analgesic, Pain

\section{INTRODUCTION}

Tramadol is a synthetically derived analgesic drug with two isomeric forms and has a double action mechanism. O-desmethyl tramadol is its prime metabolite which acts as an agonist on the $\mu$-opioid receptors and has a feeble affinity. The synergistic way of action on pain transmission mechanisms is through its monoaminergic activity. To be precise, tramadol hampers re-uptake of serotonin and noradrenaline by neurons which stimulates the release of 5 -HT, thus intensifying spinal pain inhibitory pathways. ${ }^{1}$ Subjects with tramadol overdose commonly present with respiratory and central nervous system depression. ${ }^{2}$ Here, we present the case of a 7 -year-old boy, who along with loss of consciousness and slowed breathing, presented with decreased blood sugar levels.

\section{CASE REPORT}

A 7-year-old boy was referred to our clinic in an unconscious state. On medical history recording, the parents of the subject reported that he had accidentally ingested 7 tablets of tramadol approximately 45 minutes before the presentation. This was considered to be an unintentional intoxication of a child at home due to negligence. He vomited a few times at home and ultimately lost his consciousness.

On examination, he showed clamminess, low blood pressure $(87 / 59 \mathrm{~mm} \mathrm{Hg})$, was pale and unconscious with Glasgow coma scale score of 5 , had contracted pupils, respiratory rate of $17 / \mathrm{min}$ with decreased oxygen saturation and the pulse rate of 107 beats per minute. Blood sugar levels were found to be low at 1.7 millimoles/litre. Cerebral MRI and an EEG were also non-conclusive. ECG as well as other laboratory investigations including blood cell count, electrolytes, urea and creatinine and liver function tests showed normal values.

An injection of naloxone was given as an antidote which led to a quick improvement. Intravenous dextrose infusion was given to treat low blood sugar and hypoxia was corrected using oxygen. He regained consciousness few hours after the admission. He was discharged home next day with favourable outcome but did not return for follow-up. His condition was confirmed on a telephonic conversation with his father and was found to be stable.

\section{DISCUSSION}

Tramadol is an opioid synthetic pain killer acting centrally and is commonly used for management of moderate to severe grade pain, but not often recommended for children because its safety profile for low age group has not yet been established. The suggested dosage for therapeutic purpose in children stands at $1-2 \mathrm{mg} / \mathrm{kg} 6$ hourly. The peak level of this drug in serum is achieved in half an hour and it is eventually eliminated renally. Serum therapeutic levels range from 0.2 to $0.8 \mathrm{mg} / \mathrm{L}$, and toxicity is considered when it goes above $1 \mathrm{mg} / \mathrm{L}^{3,4}$

Till date, only a few cases of tramadol poisoning have been reported in medical literature. ${ }^{5}$ According to the International Association of Forensic Toxicologists, respective blood levels in adults representing therapeutic, toxic and lethal doses ranges from 0.1 to $0.8 \mathrm{mg} / \mathrm{L}, 1-2 \mathrm{mg} / \mathrm{L}$ and greater than $2 \mathrm{mg} / \mathrm{L}^{6}{ }^{6}$ 
Tramadol hydrochloride usually affects respiratory system and central nervous system producing a wide constellation of symptoms including extreme drowsiness, somnolence, slowed breathing and heartbeat, nausea, vomiting, clamminess, contraction of pupils, constipation, sweating, muscle weakness, seizures, pruritus and lethargy similar to those of other opioids. Literature reports have shown that tramadol related seizures are dose dependent. However, direct association between blood concentration to occurrence or severity of seizure has not been documented. ${ }^{7}$ When used concomitantly with drugs that hamper tramadol mechanism or serotonergic medicines, potentially life-threatening or fatal serotonin syndrome may occur. Decreased blood sugar level has been reported in a few cases, however, it is an unexpected presentation of tramadol intoxication. ${ }^{8,9}$ Cheng JT et al. in a study on rats in 2001, showed that activation of $\mu$-opioid receptors, which is the primary target of tramadol found mainly in the central nervous system and hepatocytes results in an enhanced utilization of glucose utilization and reduction of hepatic gluconeogenesis resulting in hypoglycemia. ${ }^{10}$ Moreover, a few cases of hypoglycemia induced by selective serotonin re-uptake inhibitors (SSRI's) have been reported. ${ }^{11}$

Tramadol should be avoided with alcohol, CNS depressants and other opioids. Treatment should be focused on adequate ventilation. In the present case, nature of the treatment was mainly conservative. However, our patient was closely monitored, and he showed a remarkable recovery without any neurological complications.

\section{CONCLUSION}

Physicians and healthcare professionals should be aware of the fact that although not very common, acute tramadol intoxication may lead to a decreased blood glucose level. Tramadol overdose can be lifethreatening or even fatal if not handled appropriately, so no delay should be made in recognizing its symptoms.

\section{REFERENCES}

1. Schug SA, Ffpmanzca F, Lim A. Tramadol, Prescriber Update 2000;20:26-30.

2. Shadnia S, Soltaninejad K, Heydari K, Sasanian G, Abdollahi M. Tramadol intoxication: A review of 114 cases. Hum Exp Toxicol 2008;27:201-5.

3. Grond S, Sablotzki A. Clinical pharmacology of tramadol. Clin Pharmacokinet. 2004;43(13):879923.

4. Saudan S, Habre W. Pharmacokinetics of tramadol in children. Ann Fr Anesth Reanim. 2007;26:560-563.

5. Hassanian-Moghaddam H, Farnaghi F, Rahimi M. Tramadol overdose and apnea in hospitalized children: A review of 20 cases. Res Pharm Sci. 2015; 10(6): 544-552.

6. Pothiawala S, Ponampalam R. Tramadol overdose: a case report. Proc Singap Healthc. 2011;20:219-3.

7. Taghaddosinejad F, Mehrpour O, Afshari R, Seghatoleslami A, Abdollahi M, Dart RC. Factors related to seizure in tramadol poisoning and its blood concentration. J Med Toxicol 2011;7:183-8.

8. Tramadol Toxicity and Treatment, Toxic Update BC Drug and Poison Information Centre. 2010;6. Available from: http://www.dpic.org/sites/default/files/10 v6 in $T$ ramadol.pdf. [Last accessed on 2018 Sept 15].

9. Mugunthan N, Davoren P. Danger of hypoglycemia due to acute tramadol poisoning. Endocr Pract 2012;18:e151-2.

10. Cheng JT, Liu IM, Chi TC, Tzeng TF, Lu FH, Chang CJ. Plasma glucose-lowering effect of tramadol in streptozotocin-induced diabetic rats. Diabetes 2001;50:2815-21.

11. Bourne C, Gouraud A, Daveluy A, Grandvuillemin A, Auriche P, Descotes J, et al. Tramadol and hypoglycaemia: comparison with other step 2 analgesic drugs. Br J Clin Pharmacol 2013;75:1063-7. 